\title{
Spatial-Temporal Distribution and Key Drivers of Inequalities in the Gap Between Health-Adjusted Life Expectancy and Life Expectancy: Evidence from a Megacity in China, 2010-2019.
}

\author{
Junyan Xi \\ Sun Yat-Sen University \\ Yuanyuan Chen \\ Guangzhou Center for Disease Control and Prevention \\ Yuqin Zhang \\ Sun Yat-Sen University \\ Ao Luo \\ Guangdong Center for Disease Control and Prevention \\ Zhicheng Du \\ Sun Yat-Sen University \\ Boheng Liang \\ Guangzhou Center for Disease Control and Prevention \\ Hang Dong \\ Guangzhou Center for Disease Control and Prevention \\ Xiao Lin \\ Sun Yat-Sen University \\ Pengzhe Qin \\ Guangzhou Center for Disease Control and Prevention \\ Yuantao Hao ( $\nabla$ haoyt@mail.sysu.edu.cn ) \\ Sun Yat-Sen University https://orcid.org/0000-0001-8024-5312
}

\section{Research}

Keywords: Health inequalities, Health-adjusted life expectancy, Megacities, Quality of life, Spatial-temporal distribution, Urban expansion Posted Date: September 29th, 2021

DOI: https://doi.org/10.21203/rs.3.rs-934308/v1

License: (1) This work is licensed under a Creative Commons Attribution 4.0 International License. Read Full License 


\section{Abstract}

Purpose The improvement of the quality of life (QoL) is reflected in the narrowing of the GAP between health-adjusted life expectancy (HALE) and life expectancy (LE). Less is known about the effect that the expansion of megacities may have on narrowing the gap. The purpose of this study was to disclose this potential relationship.

Methods Annual life tables were constructed from identified death records and population counts from multiple administrative sources

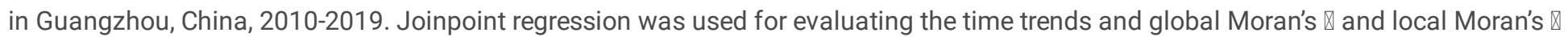
was used for evaluating spatial autocorrelation at the county-level during the entire study period. Generalized principal component analysis and multilevel models were used to examine the county-level association between the GAP and socioeconomic, health services, population structure, and population dynamics.

Results From 2010 to 2019, LE increased by 2.8 years (2.8 years for males and 2.9 years for females) and total HALE increased by 2.4 years (2.5 years for males and 2.2 years for females). The counties with higher or lower LE, HALE, and their GAP were presented spatial agglomeration distribution characteristics. The central city and the neighboring counties had higher LE and HALE, and lower GAP. The ratio of male to female and the natural population growth rate were positively correlated with the GAP, while the gross domestic product per area, the density of resident population, the number of practicing (assistant) physicians per thousand, the number of hospital beds per thousand, the per capita disposable income, the ratio of child to old people, the net migration rate and the total migration rate were negatively correlated with the GAP.

Conclusion In megacities, LE and HALE were higher and their GAP was lower in the central city than in the urban fringe. Socio-economic and health services were key to narrow the GAP. The widening GAP can be explained by a high proportion of females and old people, who were longevity but unhealthy. Population density within the urban carrying capacity produced a positive effect on narrow the GAP, and these were attributed to the healthy immigrant effect.

\section{Introduction}

Advances in public health and rapid developments in socio-economic and medical technology have brought a dividend of life $\mathrm{e}^{1,2}$, with steady improvements in global health over the past 30 years $^{3}$. Global Life Expectancy (LE) at birth rosed to 73.5 years in 2019 from 67.2 years in $2000^{4}$. However, longer survival does not equate to a better Quality of Life $(\mathrm{QoL})^{5}$. After accounting for population growth and aging, chronic non-communicable diseases carry a greater burden than acute infectious diseases, and the pattern of disability shifts from traditional congenital disability or physical disorders to mental and behavioral disorders ${ }^{6}$. A large number of medical resources are consumed by non-lethal chronic non-communicable diseases and disabilities, while LE does not fully reflect their impact on the health of the population. That is, the LE is no longer able to comprehensively evaluate the health of the human life cycle or to assess the effectiveness of health interventions strategies. To measure how long a population can live without disease or disability, the concept of Healthy Life Expectancy (HLE) has been proposed ${ }^{7}$. HLE is divided into Health State Expectancy (HSE) and Health-Adjusted Life Expectancy (HALE) ${ }^{8}$. HSE is not a comprehensive measure of health because it only focuses on survival time in a specified state of health (such as without disability and being able to live independently). HALE compensates for the limitations of HSE by weighting all causes and sequelae based on LE. HALE has been used as a comprehensive indicator for monitoring changes in the health of populations in the Global Burden of Diseases Study (GBD). Globally, HALE at birth increased from 58.6 years in 2000 to 63.5 years in $2019^{4}$. The difference between LE and HALE reflects the number of years populations have lived in a state of diseases or disability, a larger difference indicates a longer life in an unhealthy state and lower QoL. Not only improving LE and HALE but also focusing on narrowing their gap, is the goal of the global health field. Some studies have mentioned the gap and called the indicator LE-HALE ${ }^{9,10}$, but there are few in-depth analyses of $\mathrm{it}^{11}$, particularly whether the phase of unprecedented urbanization since the 20 th century has narrowed it.

Megacities are defined as cities with more than 10 million inhabitants ${ }^{12}$. Globally, the number of megacities is projected to rise from 33 in 2018 to 43 in $2030^{12}$. The rapid growth in the number of megacities and the huge population size of existing megacities will create a large number of environmental and social problems, which will reduce the QoL and create great challenges and opportunities for sustainable development in the future ${ }^{13-17}$. While focusing on the related factors that can explain health inequalities will provide important information for realizing more equitable population health ${ }^{18-22}$, less is known about the effect that the expansion of megacities may have on narrowing the gap between LE and HALE (GAP). Besides, local characteristics were not taken into account in 
previous studies on the relationship between health inequalities and socioeconomic levels ${ }^{23}$. China which houses six of the world's 33 megacities and more than any other country, may provide compelling evidence. Deepening Reform of Medicine and Healthcare System was launched in China in 2009, to make China capable of providing basic medical services to all its citizens by $2020^{24,25}$. This follows several rounds of reform in the Chinese medicine and healthcare system, with remarkable achievements and rapid transition of the health of Chinese citizens ${ }^{26}$. In China, LE at birth rosed to 77.6 years in 2019 from 68.1 years in 1990, and HALE at birth increased to 68.4 years in 2019 from 60.4 years in $1990^{4}$. Guangzhou is one of the six megacities in China and a very important world city that links major economic regions and states into the world economy ${ }^{27}$, known as China's southern gate to the world. As a representative international metropolis at the forefront of reform, Guangzhou has become the epitome of China. From 1978 to 2015, the annual growth rate of urban expansion in Guangzhou reached $8.07 \%$, ranking second among the six megacities in China ${ }^{28}$. During the 35 years from 1979 to 2013, this megacity experienced four urban expansion stages (low rates, increased rates, high rates, and steadily increased rates) and three urban growth models (edge-expansion growth, infilling growth, and spontaneous growth), and is still developing, which has had a significant impact on the urban spatial pattern and the direction of urban development ${ }^{29}$. Examining LE, HALE, and their GAP by county allowing for tracking spatial-temporal distribution and assessing factors related to these disparities, which has important implications. In particular, policies and programs that aiming to achieve healthy and sustainable future cities have the potential to narrow the GAP in all locations, especially those currently most disadvantaged, consequently reducing health inequalities.

In the light of the above, this study mainly has the two specific objectives as follows. Firstly, to generate annual estimates of LE, HALE, and their GAP of all districts of Guangzhou, China, 2010-2019, and analyze their spatial-temporal distribution. Secondly, to assess the extent to which variation in the GAP is explained by variation in socio-economic, health services, population structure, and population dynamics.

\section{Methods}

\section{Study Area and Data Sources}

Guangzhou is located in the south of China, on the lower reaches of the Pearl River and near the South China Sea. It covers an area of $7,500 \mathrm{~km}^{2}$ and is composed of 11 districts. By the end of 2019, the number of inhabitants of Guangzhou was about 15.3 million. The datasets of all-cause deaths were obtained from Guangzhou Center for Disease Control and Prevention, covering all districts of Guangzhou from 2010 to 2019. Before 2015, the divisions of districts in Guangzhou were different from the present. Luogang District used to be independent and has been merged into Huangpu District. Therefore, we combined the datasets of Luogang and Huangpu District from 2010 to 2014. The datasets of all-cause deaths were mainly based on the death cases reported by the Chinese National Cause of Death Registration and Reporting Information System and integrates the data from the Statistics Information Center of Guangzhou Health Bureau, Guangzhou Public Security Bureau, and Maternal and Child Health Department. All of the causes of death were originally coded by the International Classification of Diseases (ICD-10), with duplicate data cleared. The cases with logic errors were reviewed one by one, the root cause of death was verified according to the multiple causes of death and demographic information, and the ICD-10 code was corrected and confirmed. The registered population datasets from 2010 to 2019 were obtained from the Guangzhou Public Security Bureau. The indexes of socio-economic, health services, population structure, and population dynamics of all districts of Guangzhou were obtained from the Statistical Yearbook of Guangzhou from 2011 to 2020 published by the Guangzhou Statistics Bureau ${ }^{30}$. Open-access data of GBD is available from the Global Health Data Exchange website ${ }^{31}$. This study complies with the Guidelines for Accurate and Transparent Health Estimates Reporting (GATHER) statement.

\section{Life expectancy, health-adjusts life expectancy, and their GAP}

Abridged life tables for 2010-2019, which were prepared by previous research ${ }^{32}$, were calculated for the 19 standard age groups by sex. The first two age groups in the abridged life table are $<1$ years and 1-4 years. After that, the age groups are divided according to the standard of five years, and the last age group is > 85 years, which is open. Method to estimating HALE was developed by GBD and WHO, and details are in the appendix. Data management and compilation of annual life tables to calculate LE, HALE, and their GAP was completed using Microsoft Excel 2019 (Microsoft Corporation, Redmond, Washington).

\section{Spatial-temporal analysis}


JoinPoint regression analysis was used to estimate the Annual Percentage Change (APC), the Average Annual Percentage Change (AAPC), and the corresponding 95\% Confidence Interval (Cl) for each trend segment of LE and HALE during the 10-year study period to examine temporal trends ${ }^{33}$. The analysis was conducted using the Joinpoint trend analysis software, version 4.8.0.1 (National Cancer Institute, USA). To evaluate the spatial distribution patterns of median LE, HALE, and their GAP during the 10-year study period, the

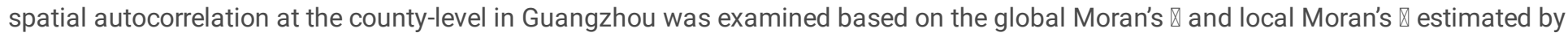
R software, version 4.1.0 (R Foundation for Statistical Computing, Vienna, Austria). The spatial distribution maps were drawn by using ArcGIS software, version 10.8.1 (Environmental Systems Research Institute Inc, Redlands, CA), and GeoDa software, version 1.16.0.16 (University of Chicago Center for Spatial Data Science, USA).

\section{Correlation analysis}

A multilevel model, with the annual county-level GAP of all districts of Guangzhou as the dependent variable from 2010 to 2019 , was used to analyze the direction and strength of the relationship between the GAP and socio-economic, health services, population structure, and population dynamics. Panel datasets of all districts of Guangzhou from 2010 to 2019 are composed of original variables covering the above four dimensions, which are considered the general survey main indicators of development level for China, covering the above four dimensions, and new variables are calculated based on the original variables: a) Ratio of male to female = number of male / number of female; $b$ ) Ratio of child to old people = number of young people aged 14 and below / number of old people aged 60 and above; c) Natural population growth rate = birth rate - death rate; d) Gross domestic product per area $=$ gross domestic product $/$ district area; $\mathrm{e}$ ) Total migration rate = migration rates + emigration rate; f) Net migration rate = migration rates emigration rate. Kaiser-Meyer-Olkin test and Bartlett's test were used to test for collinearity between variables. Generalized Principal Component Analysis (GPCA) ${ }^{34}$ was used for 10 consecutive years of panel datasets, which effectively avoids the deviation of results caused by short-term fluctuations and is more robust and representative, and principal components with eigenvalues greater than one were selected as independent variables of the multilevel model. The Akaike Information Criterion (AIC) and Bayesian Information Criterions (BIC) were used to select the optimal model. For all models, parameter estimates and standard errors for fixed and random coefficients, as well as conditional $R^{2}$ and marginal $R^{2}$, were reported. The correlation coefficients of the original variables can be obtained through the reverse derivation of the correlation coefficients of the principal component, which is based on the theory that the principal component is a linear combination of the original variables.

$$
\beta^{\prime}{ }_{i}=\left(\begin{array}{lll}
\beta_{1} & \cdots & \beta_{m}
\end{array}\right)\left(\begin{array}{c}
\sqrt{\lambda_{1}} \times \alpha_{i 1} \\
\vdots \\
\sqrt{\lambda_{m}} \times \alpha_{i m}
\end{array}\right)
$$

$\beta^{\prime}$ is the correlation coefficient of the original variable, $\beta$ is the correlation coefficient of the principal component, $\lambda$ is the eigenvalues of the principal component, and $a$ is the factor loading of the original variable. $i$ is the number of original variables, and $m$ is the number of principal components.

\section{Results}

\section{The district-specific LE, HALE, and their GAP in Guangzhou, 2019}

In 2019, the LE, HALE, and their GAP of females in Guangzhou were higher than that of males. In the 11 districts of Guangzhou, the LE of 10 districts were over 80 years, and the HALE of 7 districts were over 75 years (Table 1). LE and HALE showed a higher level in the central city and a lower level in the urban fringe. On the contrary, the GAP showed a lower level in the central city and a higher level in the urban fringe (Fig.1).

Table 1 Estimates of district-specific LE, HALE, and their GAP in Guangzhou, 2019 


\begin{tabular}{|lllllllllll|}
\hline Districts & LE & & \multicolumn{3}{c|}{ HALE } & \multicolumn{3}{c|}{ GAP } \\
\cline { 2 - 6 } & Males & Females & Total & Males & Females & Total & Males & Females & Total \\
\hline Liwan & 80.0 & 86.4 & 83.1 & 73.9 & 78.4 & 76.0 & 6.1 & 8.0 & 7.1 \\
\hline Yuexiu & 82.0 & 87.3 & 84.6 & 76.6 & 79.1 & 77.9 & 5.4 & 8.2 & 6.7 \\
\hline Haizhu & 80.6 & 86.1 & 83.3 & 74.9 & 77.7 & 76.3 & 5.7 & 8.4 & 7.0 \\
\hline Tianhe & 83.0 & 88.0 & 85.4 & 78.0 & 80.2 & 79.1 & 5.0 & 7.8 & 6.3 \\
\hline Baiyun & 80.2 & 85.3 & 82.7 & 74.5 & 76.5 & 75.7 & 5.7 & 8.8 & 7.0 \\
\hline Huangpu & 79.6 & 85.3 & 82.4 & 73.5 & 76.6 & 75.0 & 6.1 & 8.7 & 7.4 \\
\hline Panyu & 80.3 & 86.7 & 83.5 & 74.4 & 78.8 & 76.7 & 5.9 & 7.9 & 6.8 \\
\hline Huadu & 79.4 & 84.9 & 82.1 & 73.4 & 75.9 & 74.7 & 6.0 & 9.0 & 7.4 \\
\hline Nansha & 77.2 & 84.3 & 80.7 & 69.8 & 74.8 & 72.2 & 7.4 & 9.5 & 8.5 \\
\hline Conghua & 75.8 & 83.1 & 79.3 & 68.0 & 73.0 & 70.2 & 7.8 & 10.1 & 9.1 \\
\hline Zengcheng & 77.9 & 84.0 & 80.8 & 70.9 & 74.6 & 72.6 & 7.0 & 9.4 & 8.2 \\
\hline Total & 80.1 & 85.9 & 82.9 & 74.0 & 77.3 & 75.6 & 6.1 & 8.6 & 7.3 \\
\hline
\end{tabular}

$\mathrm{LE}=$ life expectancy. HALE = health-adjusted life expectancy. GAP = the difference between LE and HALE.

\section{Temporal trends of LE and HALE in Guangzhou from 2010 to 2019}

From 2010 to 2019, the LE and HALE in Guangzhou showed a steady increasing trend. Total LE increased by 2.8 years and AAPC was 0.4 ( $95 \% \mathrm{Cl}: 0.3-0.4)$, the LE of males increased by 2.8 years and AAPC was $0.4(95 \% \mathrm{Cl}: 0.3-0.5)$, and the LE of females increased by 2.9 years and AAPC was 0.4 (95\% Cl: 0.3-0.4). Total HALE increased by 2.4 years and AAPC was 0.3 (95\% Cl: 0.3-0.4), the HALE of males increased by 2.5 years and AAPC was 0.4 (95\% Cl: $0.3-0.5)$. The HALE of females increased by 2.2 years with AAPC and 0.3 (95\%Cl: $0.2-$ 0.4), including APC for 2010 to 2017 was 0.2 (95\%Cl: 0.1-0.3) and APC for 2017 to 2019 was 0.6 (95\%Cl: -0.1-1.3) (Fig.2).

\section{Spatial autocorrelation for LE, HALE, and their GAP in Guangzhou from 2010 to 2019}

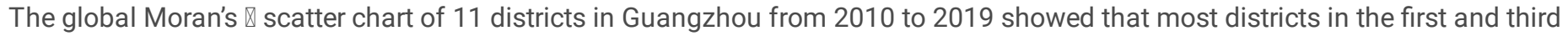
quadrants, indicating that districts with higher or lower LE, HALE, and their GAP were presented spatial agglomeration distribution characteristics (Fig.3). Further, the existence of local spatial autocorrelation for LE, HALE, and their GAP was verified using the local

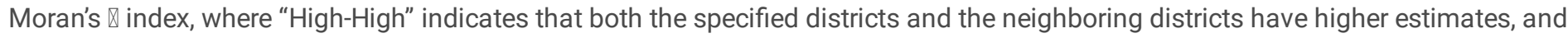
"Low-Low" indicates that both of them have lower estimates. The Local Indicators of Spatial Association (LISA) map showed that the central city, include Haizhu, Tianhe, and Yuexiu District, were "High-High" in LE and HALE and "Low-Low" in GAP, while Huadu District was "Low-Low" in HALE (Fig.4).

The relationship between the GAP and socio-economic, health services, population structure, and population dynamics in Guangzhou, 2010-2019

The null model (Model $₫$ ) with the GAP as the dependent variable was fitted, and the Intra-Class Correlation (ICC) was 0.843 , indicating that $84.3 \%$ of the total variation in the GAP was attributed to districts. That is, there was an interclass correlation and the multilevel

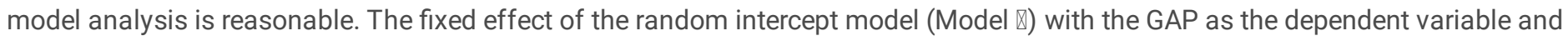
time as the independent variable showed the GAP increasing by 0.050 years per year. In the models with random intercept and slope

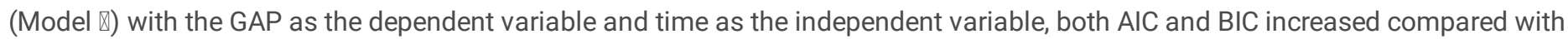

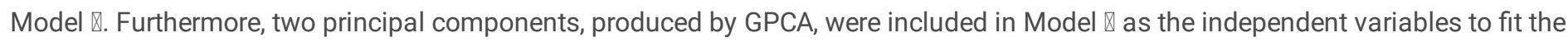
multilevel model (Model $\varangle$ ) with a controlled principal component based on Model $\varangle$. The results showed that the fixed effect and random effect could explain $83.8 \%$ of the variation in the GAP, and the GAP increasing by 0.092 years per year (Table 2).

Table 2 Parameter estimates of mixed effect model (standard error) 


\begin{tabular}{|c|c|c|c|c|}
\hline Parameter & Model $\rrbracket^{a}$ & Model $\rrbracket^{\mathrm{b}}$ & Model $\nabla^{c}$ & Model $\rrbracket^{d}$ \\
\hline \multicolumn{5}{|c|}{ Fixed Parameters } \\
\hline (Intercept) & $7.353(0.255)^{\star \star \star}$ & $7.076(0.262)^{\star \star \star}$ & $7.077(0.250)^{\star \star \star}$ & $6.846(0.209)^{\star \star \star}$ \\
\hline (Years) & - & $0.050(0.011)^{\star \star \star}$ & $0.050(0.015)^{\star \star}$ & $0.092(0.018)^{\star \star \star}$ \\
\hline (Comp1) & - & - & - & $0.175(0.057)^{\star \star}$ \\
\hline (Comp2) & - & - & - & $0.113(0.051)^{\star}$ \\
\hline \multicolumn{5}{|c|}{ Random Parameters } \\
\hline (Districts) & $0.703(0.838)$ & $0.705(0.840)$ & $0.642(0.801)$ & $0.367(0.606)$ \\
\hline (Years) & - & - & $0.001(0.037)$ & - \\
\hline (Residual) & $0.131(0.362)$ & $0.109(0.330)$ & $0.097(0.312)$ & 0.107 (0.328) \\
\hline
\end{tabular}

$\star \star \star P<0.001, * \star P<0.01, * P<0.05$

Conditional $R^{2}=0.838 \bigotimes$ Marginal $R^{2}=0.282$

a The null model with the GAP as the dependent variable.

${ }^{\mathrm{b}}$ The random intercept model with the GAP as the dependent variable and time as the independent variable.

${ }^{\mathrm{c}}$ The random intercept and slope model with the GAP as the dependent variable and time as the independent variable.

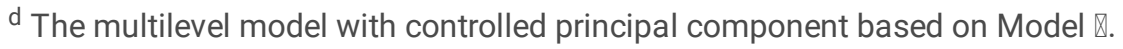

Comp1 and Comp2 = the principal component produced by generalized principal component analysis

The ratio of male to female and the natural population growth rate were positively correlated with the GAP, while the gross domestic product per area, the density of resident population, the number of practicing (assistant) physicians per thousand, the number of hospital beds per thousand, the per capita disposable income, the ratio of child to old people, the net migration rate and the total migration rate were negatively correlated with the GAP (Fig.5).

\section{Discussion}

More and more indexes are being used to define health, but the GAP between LE and HALE has received little attention. The GAP focuses on revealing the unhealthy survival time of the population, which can be used as a routine health surveillance index for tracking population health over time. No research focusing on LE, HALE, and their GAP at the Chinese city-level, but this study was based on a typical megacity that is representative in China, make up for the gap in such a field. This study assessed the extent to which variation in the GAP is explained by variation in socio-economic, health services, population structure, and population dynamics, with an added value: our results provided a reminder of the challenges policymakers will face in balancing health equality and urban expansion as the number and population size of megacities increase dramatically.

\section{Time trends from 2010 to 2019 and current status in 2019 for LE, HALE, and their GAP in Guangzhou}

In general, LE and HALE in Guangzhou increased significantly from 2010 to 2019. LE increased by 2.8 years and HALE increased by 2.4 years. The growth rate of LE was slightly higher than that of HALE, which was similar to the situation in China and its provinces ${ }^{35}$. There was a small difference in the growth rate between LE and HALE (the GAP increasing by 0.092 years per year), suggested that while the lifetime was prolonged, QoL was also increased synchronously. In 2019, LE and HALE in Guangzhou were 82.9 years (80.1 years for males and 85.9 years for females) and 75.6 years ( 74.0 years for males and 77.3 years for females), both of which were higher than the Chinese average reported by GBD $2019^{4}$. However, the health of the population is not satisfactory in all districts. The top three districts of LE and HALE are Tianhe, Yuexiu, and Panyu District, while the lower ones are Conghua, Zengcheng, and Nansha District. The differences between the highest and lowest values of LE, HALE, and their GAP were 6.1 years, 8.9 years, and 2.8 years. The wide differences implied that the positive results at the city-level mask health inequalities at the sub-regional level. 


\section{Spatial distribution characteristics for LE, HALE, and their GAP in Guangzhou from 2010 to 2019}

Our findings indicated that the GAP was smaller in the districts with higher LE and HALE, but larger in the districts with lower LE and HALE, which led to the obvious polarization of unhealthy survival time in Guangzhou. From the perspective of urban planning, the above distribution was consistent with the distribution of the central city and urban fringe. The combined effect of developed cities will lead to urban expansion, that is, the space of the central city is limited, the accommodation degree decreases, and the industries move to the urban fringe. The driving force of urban expansion is determined by socioeconomic factors and physical factors ${ }^{36}$, as evidenced by the development patterns of Guangzhou ${ }^{29}$. Particularly, socio-economic factors such as the rapid development of the tertiary industry in the Pearl River Delta region and the migration of people to work in developed areas may provide great opportunities for urban expansion in Guangzhou ${ }^{37,38}$. As a pioneer city in implementing China's urban-rural planning, Guangzhou has eliminated urban construction problems such as economic backwardness, low urbanization rate, and unreasonable regional economic development through its unique planning system ${ }^{39,40}$, which has greatly improved the LE and HALE in urban fringe and reached above the Chinese average ${ }^{4}$. However, there are still health inequalities between the central city and the urban fringe, as our study found. It is necessary for the urban-rural planning of Guangzhou to pay attention to the challenge in balancing health equality and urban expansion as urbanization accelerates.

\section{Effects of socio-economic and health services on changes in GAP}

Current analysis showed that flourishing socio-economic (gross domestic product per area, per capita disposable income) and adequate health services (number of practicing (assistant) physicians per thousand, number of hospital beds per thousand) have a positive effect on narrowing the GAP. Population health is the result of investments, such as good environmental conditions, healthy lifestyle, and appropriate medical and health services. Health service is not only an input factor to maintain national health, but also an important source of national income growth. At the same time, it also has comparative advantages in promoting employment and personal income, thus contributing to the improvement of household consumption and national domestic demand, and boosting the transformation of the economic development model ${ }^{41}$. A virtuous cycle of socioeconomic and health services is key to narrow the GAP.

\section{Effects of population structure on changes in GAP}

Our results showed a positive correlation between the ratio of male to female and GAP, which may be explained by the fact that although females' LE increased faster than males and the trend has remained at higher levels ${ }^{42}$, the increase in HALE is not parallel. It is well known that females generally live longer than males, but female's longer lives are not necessarily healthy lives ${ }^{43-45}$. This phenomenon is called the male-female health-survival paradox ${ }^{46}$. Several mechanisms have been proposed to explain the male-female health-survival paradox, include gender differences in biological risks, and risks attribute to social roles, lifestyle, and health behaviors ${ }^{47}$. Translating the longevity advantage of females into health advantage should be considered as the health policy goal of megacities.

We also found the GAP was negatively correlated with the ratio of child to old people. It is generally agreed that, although an aging population brings longer life, it does not represent an increase in health ${ }^{48}$. The health of old people should be more concerned with their functional integrity than the presence or absence of disease ${ }^{49}$. To narrow the GAP, healthy aging is urgent. China has long regarded population aging as the top priority of public health issues and has already formulated a series of policies and measures to deal with the pressure of aging society ${ }^{50,51}$. In addition, geriatrics has developed rapidly in recent years into a subdiscipline ${ }^{52}$. Attention should be paid to provide old people with high-quality prevention and care capabilities that will enable them to remain resilient and functional as they age, taking them one step closer to the goal of healthy aging.

\section{Effects of population dynamics on changes in GAP}

In terms of population dynamics, the GAP was negatively correlated with the resident population density, the net migration rate, and the total migration rate, and positively correlated with the natural population growth rate. It is not difficult to understand that regional development and economic progress must be accompanied by population aggregation ${ }^{53}$, 54 . Population aggregation to a certain extent is conducive to the integration of resources, the acquisition of social services, and the effective implementation of regional economic activities $^{55}$. However, the too high population density will also lead to the shortage of per capita resources and the increase of social burden ${ }^{56}$. In brief, the relationship between population density and health index presents an inverted U-shaped curve ${ }^{57}$. Guangzhou, with the favorable economic growth environment and the speed of infrastructure construction, has brought large numbers of migrants. It is 
generally accepted that the link between migration and health effects is different for these people with different social backgrounds. For international migrants, lack health insurance coverage, face language and cultural barriers to medical care, and fear that access to public institutions or health care providers may lead to legal problems and even possible deportation ${ }^{58}$. For internal migrants, especially rural-to-urban migrants in China faces some limitations that employment in public sectors, health insurance, and public assistance programs in cities $^{59}$. Therefore, restrictive immigration management is needed to produce a healthy immigrant effect ${ }^{60,61}$. The Chinese Household Registration System plays a two-way role in managing immigration. On the one hand, social welfare can be popularized for every citizen who legally obtains the urban household registration, and the health of the citizen who holds the household registration is promised to be guaranteed. On the other hand, the government will also dynamically limit the population expansion according to the process of urban development, so that the social support can stabilize the supply ${ }^{62}$. It can be seen that for megacities, immigration management guided by health policy is an important link to control massive population growth and narrow the GAP.

\section{Advantages and limitations}

This study has several advantages. First of all, it provides the latest evaluation of the spatial-temporal distribution of LE, HALE, and their GAP in Guangzhou for the first time, and provides important scientific evidence for global health study. Secondly, it was the first that links the GAP between LE and HALE with the expansion of megacities and provides valuable knowledge about the role of social determinants in QoL. Meanwhile, some limitations of our work should be identified. First, we cannot obtain complete and accurate information on the prevalence and disability severity for all-cause and sequelae, so YLDs could not be directly calculated like the GBD. But the well-established indirect method developed by the WHO also provides an alternative method of calculating YLDs, whose results were equally reassuring, as indicated by previous studies ${ }^{63-65}$. Second, influential factors except for the four dimensions aforementioned may also be related to health inequalities, such as education, employment, and the environment, but those potential factors were not considered in this analysis. Our work is the first step to elucidating the relationship between specific social determinants and health inequalities in the expansion of megacities and pointing toward future directions for necessary research with improved study designs.

\section{Conclusion}

In megacities, LE and HALE were higher and their GAP was lower in the central city than in the urban fringe. Socio-economic and health services were key to narrow the GAP. The widening GAP can be explained by a high proportion of females and old people, who were longevity but unhealthy. Population density within the urban carrying capacity produced a positive effect on narrow the GAP, and these were attributed to the healthy immigrant effect.

\section{Abbreviations}




\begin{tabular}{|ll|}
\hline AAPC & Average Annual Percentage Change \\
\hline AIC & Akaike Information Criterion \\
\hline APC & Annual Percentage Change \\
\hline BIC & Bayesian Information Criterions \\
\hline Cl & Confidence Interval \\
\hline GATHER & Gap between HALE and LE \\
\hline GBD & Global Burden of Diseases Study \\
\hline GPCA & Generalized Principal Component Analysis \\
\hline HALE & Health-Adjusted Life Expectancy \\
\hline HLE & Healthy Life Expectancy \\
\hline HSE & Health State Expectancy \\
\hline ICC & Intra-Class Correlation \\
\hline ICD-10 & International Classification of Diseases \\
\hline LE & Life Expectancy \\
\hline LISA & Local Indicators of Spatial Association \\
\hline QoL & Quality of Life \\
\hline
\end{tabular}

\section{Declarations}

\section{Ethics approval and consent to participate}

Not applicable.

\section{Consent for publication}

Not applicable.

\section{Availability of data and material}

The datasets analyzed during the current study are available from the corresponding author on reasonable request.

\section{Competing interests}

The authors have no conflicts of interest to disclose.

Funding: a) the Guangdong Basic and Applied Basic Research Foundation (grant 2020A1515011294, 2020A1515110230 and 2021A1515011765); b) the China Postdoctoral Science Foundation (grant 2021M693594); c) the Guangzhou Municipal Health Commission (grant No.2021-2023-12, No.20201A011054); and d) Guangzhou Municipal Science and Technology Bureau (grant 2021BRP004).

\section{Authors' contributions}

Not applicable.

\section{Acknowledgments}

We wish to thank all the participants included in the study. 


\section{References}

1. Kontis V, Bennett JE, Mathers CD, et al. Future life expectancy in 35 industrialised countries: projections with a Bayesian model ensemble. The Lancet. 2017; 389: 1323-35.

2. Vogel L. Life expectancy grows with supply of primary care doctors. CAN MED ASSOC J. 2019; 191: E347.

3. Vos T, Lim SS, Abbafati C, et al. Global burden of 369 diseases and injuries in 204 countries and territories, 1990-2019: a systematic analysis for the Global Burden of Disease Study 2019. The Lancet. 2020; 396: 1204-22.

4. Wang $\mathrm{H}$, Abbas KM, Abbasifard M, et al. Global age-sex-specific fertility, mortality, healthy life expectancy (HALE), and population estimates in 204 countries and territories, 1950-2019: a comprehensive demographic analysis for the Global Burden of Disease Study 2019. The Lancet. 2020; 396: 1160-203.

5. Galvin AE, Friedman DB, Hébert JR. Focus on disability-free life expectancy: implications for health-related quality of life. QUAL LIFE RES. 2021: 1-09.

6. Murray CJL, Barber RM, Foreman KJ, et al. Global, regional, and national disability-adjusted life years (DALYs) for 306 diseases and injuries and healthy life expectancy (HALE) for 188 countries, 1990-2013: quantifying the epidemiological transition. The Lancet. 2015; 386: 2145-91.

7. Robine JM, Ritchie K. Healthy life expectancy: evaluation of global indicator of change in population health. BMJ. 1991; 302: 45760.

8. Robine J. A New Health Expectancy Classification System. Geneva: World Health Organization, 2002.

9. van Baal PH, Hoogenveen RT, de Wit AG, et al. Estimating health-adjusted life expectancy conditional on risk factors: results for smoking and obesity. POPUL HEALTH METR. 2006; 4: 14.

10. Tokudome S, Hashimoto S, Igata A. Life expectancy and healthy life expectancy of Japan: the fastest graying society in the world. BMC Research Notes. 2016; 9: 482.

11. Cao X, Hou Y, Zhang X, et al. A comparative, correlate analysis and projection of global and regional life expectancy, healthy life expectancy, and their GAP: 1995-2025. J GLOB HEALTH. 2020; 10: 1-16.

12. United Nations, Department of Economic and Social Affairs, Population Division. The World's Cities in 2018-Data Booklet. https://www.un.org/en/development/desa/population/publications/pdf/urbanization/the_worlds_cities_in_2018_data_booklet.pdf. Accessed 12th July 2021

13. Chen G, Li X, Liu X, et al. Global projections of future urban land expansion under shared socioeconomic pathways. NAT COMMUN. 2020; 11: 537.

14. Bren D Amour C, Reitsma F, Baiocchi G, et al. Future urban land expansion and implications for global croplands. Proceedings of the National Academy of Sciences. 2017; 114: 8939-44.

15. Liu X, Wang S, Wu P, et al. Impacts of Urban Expansion on Terrestrial Carbon Storage in China. ENVIRON SCI TECHNOL. 2019; 53: 6834-44.

16. Paiva ACDE, Nascimento N, Rodriguez DA, et al. Urban expansion and its impact on water security: The case of the Paraíba do Sul River Basin, São Paulo, Brazil. SCI TOTAL ENVIRON. 2020; 720: 137509.

17. Hien PD, Men NT, Tan PM, et al. Impact of urban expansion on the air pollution landscape: A case study of Hanoi, Vietnam. SCI TOTAL ENVIRON. 2020; 702: 134635.

18. Boing AF, Subramanian SV, Boing AC. Reducing socioeconomic inequalities in life expectancy among municipalities: the Brazilian experience. INT J PUBLIC HEALTH. 2019; 64: 713-20.

19. Bilal U, Cainzos-Achirica M, Cleries M, et al. Socioeconomic status, life expectancy and mortality in a universal healthcare setting: An individual-level analysis of $>6$ million Catalan residents. PREV MED. 2019; 123: 91-94.

20. Head J, Chungkham HS, Hyde M, et al. Socioeconomic differences in healthy and disease-free life expectancy between ages 50 and 75: a multi-cohort study. EUR J PUBLIC HEALTH. 2019; 29: 267-72.

21. Zaninotto P, Batty GD, Stenholm S, et al. Socioeconomic Inequalities in Disability-free Life Expectancy in Older People from England and the United States: A Cross-national Population-Based Study. The Journals of Gerontology: Series A. 2020; 75: 906-13.

22. Dwyer-Lindgren L, Bertozzi-Villa A, Stubbs RW, et al. Inequalities in Life Expectancy Among US Counties, 1980 to 2014. JAMA INTERN MED. 2017; 177: 1003. 
23. Chetty R, Stepner M, Abraham S, et al. The Association Between Income and Life Expectancy in the United States, $2001-2014$. JAMA. 2016; 315: 1750.

24. Li L, Fu H. China's health care system reform: Progress and prospects. The International Journal of Health Planning and Management. 2017; 32: 240-53.

25. Yip W, Fu H, Chen AT, et al. 10 years of health-care reform in China: progress and gaps in Universal Health Coverage. The Lancet. 2019; 394: 1192-204.

26. Yang G, Wang Y, Zeng Y, et al. Rapid health transition in China, 1990-2010: findings from the Global Burden of Disease Study 2010. The Lancet. 2013; 381: 1987-2015.

27. Loughborough University, Geography Department. Globalization and World Cities. https://www.lboro.ac.uk/gawc/world2020t.html. Accessed 12th July 2021

28. Fei W, Zhao S. Urban land expansion in China's six megacities from 1978 to 2015. SCI TOTAL ENVIRON. $2019 ; 664: 60-71$.

29. Wu Y, Li S, Yu S. Monitoring urban expansion and its effects on land use and land cover changes in Guangzhou city, China. ENVIRON MONIT ASSESS. 2016; 188: 54.

30. Guangzhou Statistics Bureau. Guangzhou Statistical Yearbook. http://112.94.72.17/portal/querylnfo/statisticsYearbook/index. Accessed 12th July 2021

31. University Of Washington, Institute For Health Metrics And Evaluation. Global Health Data Exchange. http://ghdx.healthdata.org/gbd-results-tool. Accessed 12th July 2021

32. Eayres D. Evaluation of methodologies for small area life expectancy estimation. Journal of Epidemiology \& Community Health. 2004; 58: 243-49.

33. Clegg LX, Hankey BF, Tiwari R, et al. Estimating average annual per cent change in trend analysis. STAT MED. $2009 ; 28: 3670-82$.

34. Abdi H, Williams LJ, Valentin D. Multiple factor analysis: principal component analysis for multitable and multiblock data sets. Wiley Interdisciplinary Reviews: Computational Statistics. 2013; 5: 149-79.

35. Zhou MG, Li YC, Wang HD, et al. Analysis on life expectancy and healthy life expectancy in China, 1990-2015. Zhonghua liu xing bing xue za zhi. 2016; 37: 1439-43.

36. Li M, Zhang G, Liu Y, et al. Determinants of Urban Expansion and Spatial Heterogeneity in China. INT J ENV RES PUB HE. 2019; 16: 3706.

37. Shi G, Shan J, Ding L, et al. Urban Road Network Expansion and Its Driving Variables: A Case Study of Nanjing City. INT J ENV RES PUB HE. 2019; 16: 2318.

38. Wu R, Li Z, Wang S. The varying driving forces of urban land expansion in China: Insights from a spatial-temporal analysis. SCI TOTAL ENVIRON. 2021; 766: 142591.

39. Xue D, Huang G, Guan J, et al. Changing concepts of city and urban planning practices in Guangzhou (1949-2010): An approach to sustainable urban development. CHINESE GEOGR SCI. 2014; 24: 607-19.

40. Ming LI, Yue S, Rong LU, et al. Latest Development of Village Planning in Guangzhou City: Perspectives of Urban Rural Integration. Urban Planning Forum. 2014; z1: 1-09.

41. Peking University, China Center For Economic Research. National health and economic prosperity-Based on the inspiration of the development of APEC economies. https://www.ccer.pku.edu.cn/yjcg/yjbg/243126.htm. Accessed 12 th July 2021

42. Kunitz SJ. Sex, race and social role-history and the social determinants of health. INT J EPIDEMIOL. 2007; 36: 3-10.

43. Luy M, Minagawa Y. Gender gaps--Life expectancy and proportion of life in poor health. HEALTH REP. 2014; 25: 12-19.

44. Rueda-Salazar S, Spijker J, Devolder D, et al. The contribution of social participation to differences in life expectancy and healthy years among the older population: A comparison between Chile, Costa Rica and Spain. PLOS ONE. 2021 ; 16 : e248179.

45. Van Oyen H, Cox B, Jagger C, et al. Gender gaps in life expectancy and expected years with activity limitations at age 50 in the European Union: associations with macro-level structural indicators. EUR J AGEING. 2010; 7: 229 -37.

46. Oksuzyan A, Juel K, Vaupel JW, et al. Men: good health and high mortality. Sex differences in health and aging. AGING CLIN EXP RES. 2008; 20: 91-102.

47. Oksuzyan A, Brønnum-Hansen H, Jeune B. Gender gap in health expectancy. EUR J AGEING. 2010; 7: $213-18$.

48. Huber M, Knottnerus JA, Green L, et al. How should we define health? BMJ. 2011; 343: d4163. 
49. Beard JR, Officer A, de Carvalho IA, et al. The World report on ageing and health: a policy framework for healthy ageing. The Lancet. 2016; 387: 2145-54.

50. Fang EF, Scheibye-Knudsen M, Jahn HJ, et al. A research agenda for aging in China in the 21 st century. AGEING RES REV. 2015; 24: 197-205.

51. Fang EF, Xie C, Schenkel JA, et al. A research agenda for ageing in China in the 21st century (2nd edition): Focusing on basic and translational research, long-term care, policy and social networks. AGEING RES REV. 2020; 64: 101174.

52. Fried LP, Rowe JW. Health in Aging - Past, Present, and Future. NEW ENGL J MED. 2020; 383: 1293-96.

53. Bauch CT. Wealth as a source of density dependence in human population growth. OIKOS. 2008; 117: 1824-32.

54. Population Division Of The Department Of Economic, Social Affairs Of The United Nations Secretariat. 2019 Revision of World Population Prospects. https://population.un.org/wpp/. Accessed 12th July 2021

55. Hathi P, Haque S, Pant L, et al. Place and Child Health: The Interaction of Population Density and Sanitation in Developing Countries. DEMOGRAPHY. 2017; 54: 337-60.

56. DeLong JP, Burger O. Socio-Economic Instability and the Scaling of Energy Use with Population Size. PLOS ONE. 2015; 10: e130547.

57. Wenqian F. Study on the Calculation of Healthy City Index and Its Influencing Factors. China University of Mining and Technology. 2019

58. Galea S, Freudenberg N, Vlahov D. Cities and population health. SOC SCI MED. 2005; 60: 1017-33.

59. Yi Y, Liao Y, Zheng L, et al. Health Selectivity and Rural-Urban Migration in China: A Nationwide Multiple Cross-Sectional Study in 2012, 2014, 2016. INT J ENV RES PUB HE. 2019; 16: 1596.

60. McDonald JT, Kennedy S. Insights into the 'healthy immigrant effect': health status and health service use of immigrants to Canada. Social science \& medicine (1982). 2004; 59: 1613-27.

61. Newbold KB, Danforth J. Health status and Canada's immigrant population. Social science \& medicine (1982). 2003; 57: $1981-95$.

62. Mou J, Griffiths SM, Fong HF, et al. Defining migration and its health impact in China. PUBLIC HEALTH. 2015; 129: 1326-34.

63. Menon GR, Singh L, Sharma P, et al. National Burden Estimates of healthy life lost in India, 2017: an analysis using direct mortality data and indirect disability data. The Lancet Global Health. 2019; 7: e1675-84.

64. Wen X, Sun A, Peng B, et al. Study on burden of disease in Chongqing, 2010-2013. Zhonghua liu xing bing xue za zhi. 2015; 36: 1134-38.

65. Schopper D, Pereira J, Torres A, et al. Estimating the burden of disease in one Swiss canton: what do disability adjusted life years (DALY) tell us? INT J EPIDEMIOL. 2000; 29: 871-77.

\section{Figures}



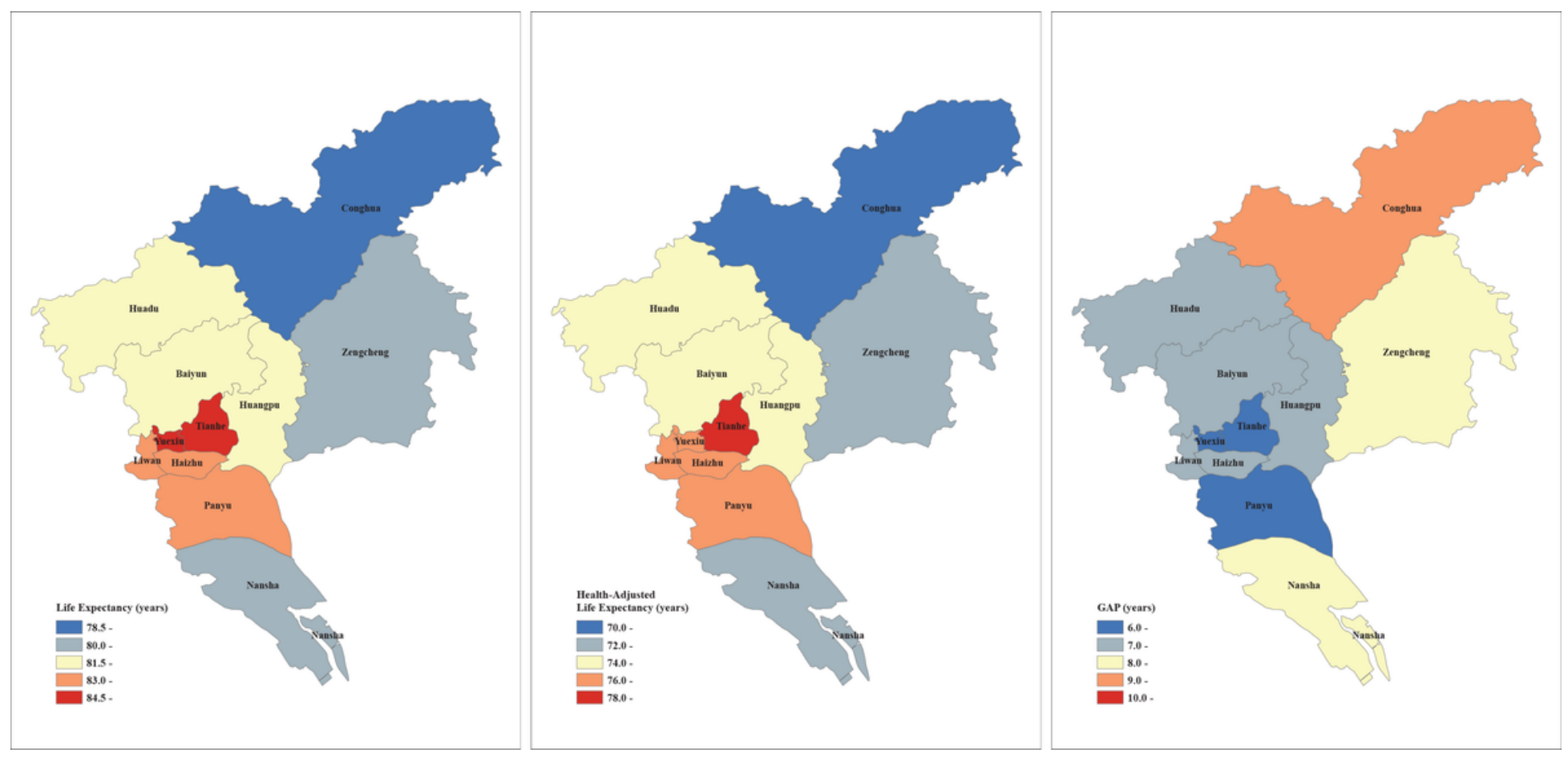

\section{Figure 1}

Spatial distribution of district-specific LE, HALE, and their GAP in Guangzhou, 2019 LE = life expectancy. HALE = health-adjusted life expectancy. GAP = the difference between LE and HALE

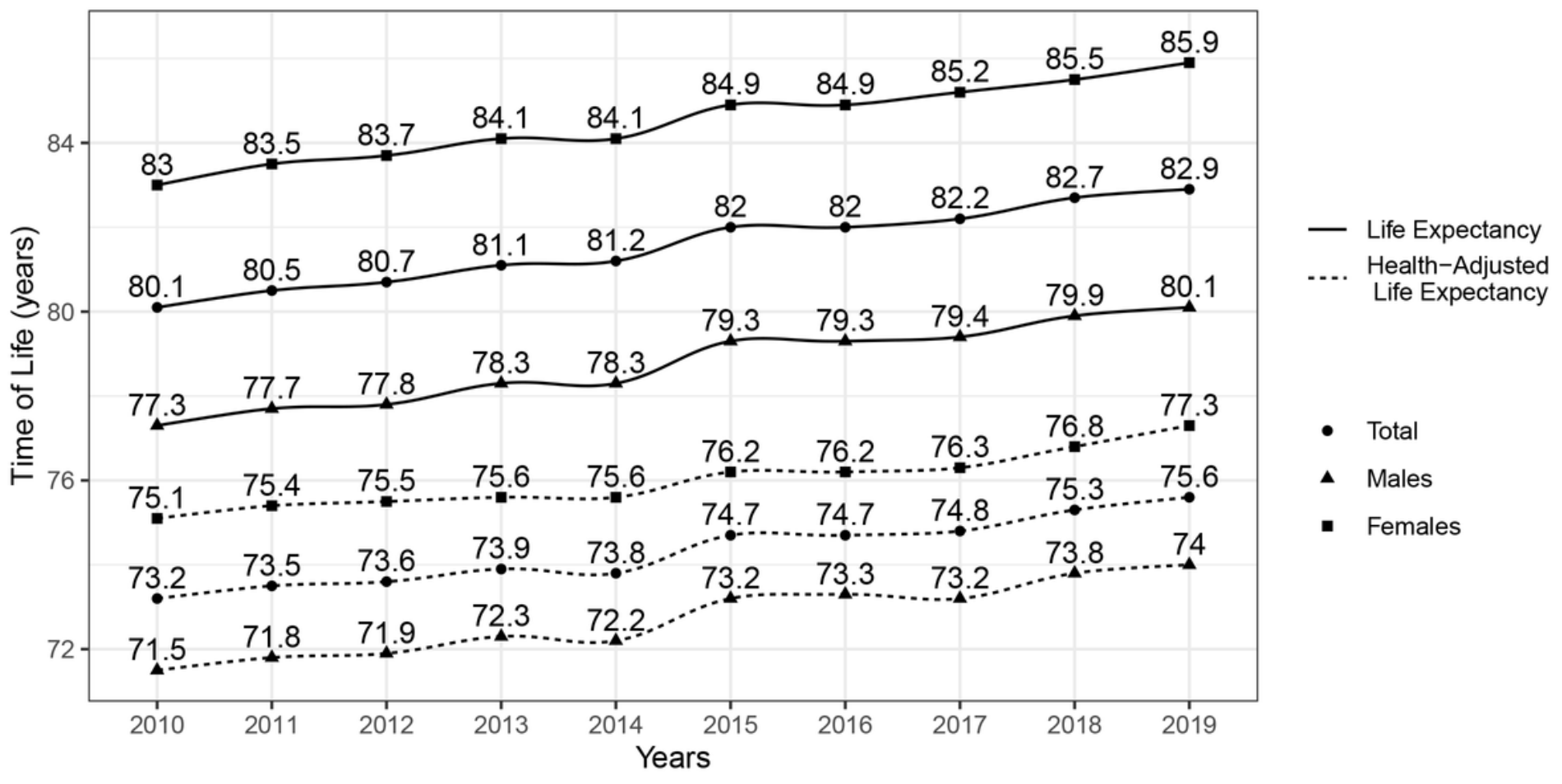

Figure 2

Temporal trends of LE and HALE in Guangzhou from 2010 to 2019 LE = life expectancy. HALE = health-adjusted life expectancy. GAP = the difference between LE and HALE. 

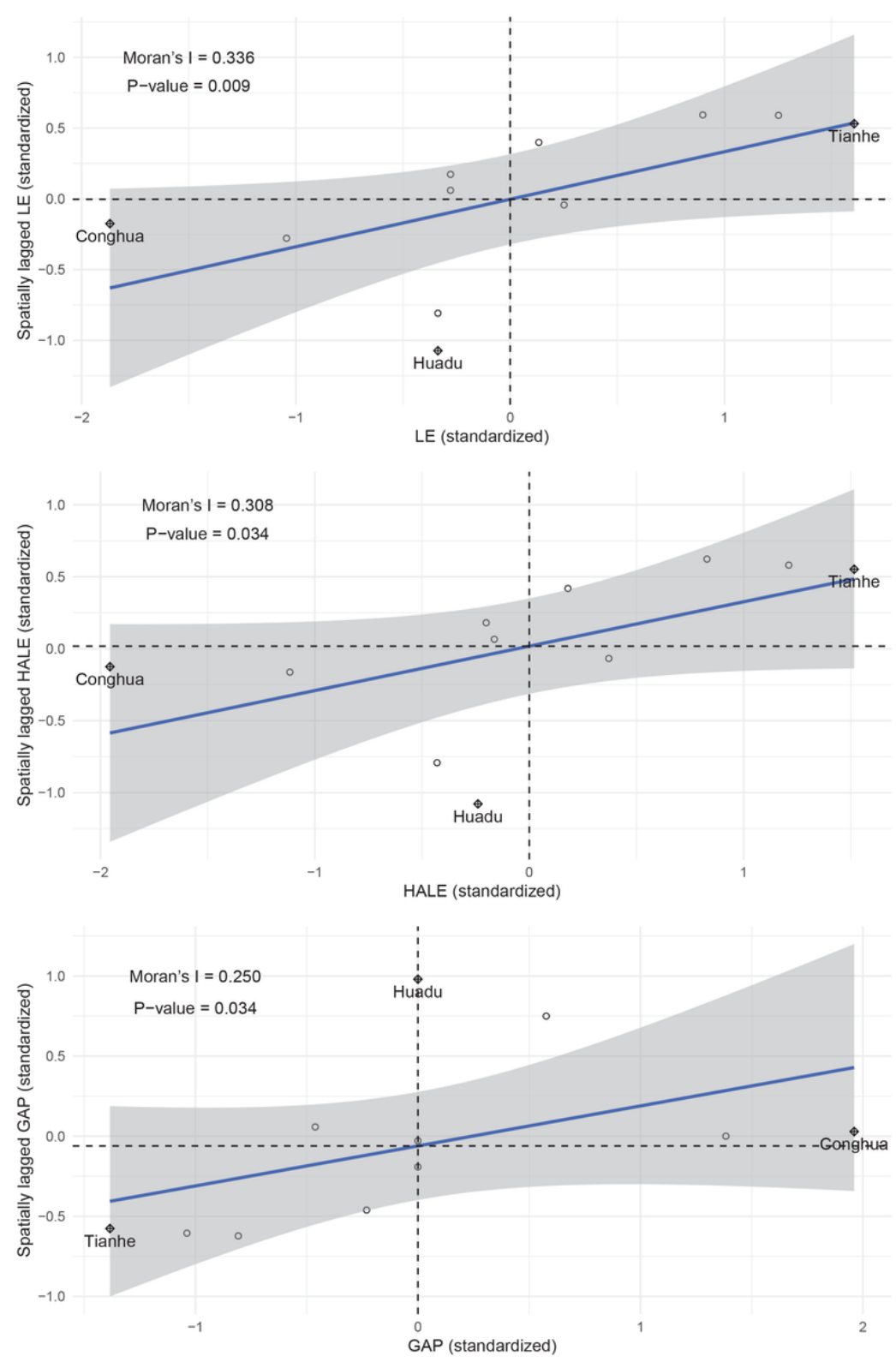

\section{Figure 3}

Global spatial autocorrelation for LE, HALE, and their GAP in Guangzhou from 2010 to 2019 The highlighted points mean high influence measures. LE = life expectancy. HALE = health-adjusted life expectancy. GAP = the difference between LE and HALE. 

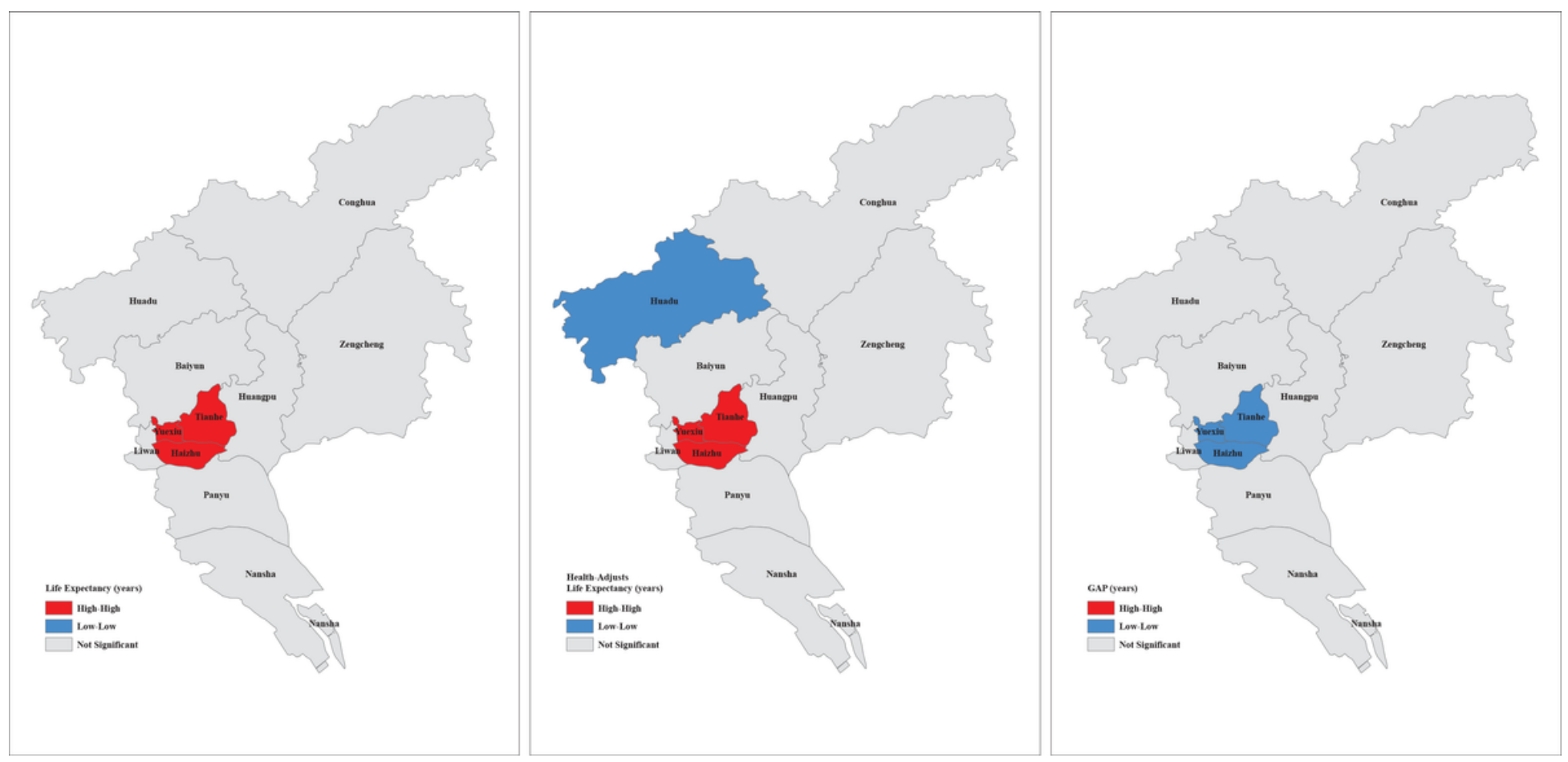

\section{Figure 4}

Local spatial autocorrelation for LE, HALE, and their GAP in Guangzhou from 2010 to 2019 High-High = both the specified districts and the neighboring districts have higher estimates. Low-Low = both the specified districts and the neighboring districts have lower estimates. $L E=$ life expectancy. HALE = health-adjusted life expectancy. GAP = the difference between LE and HALE.

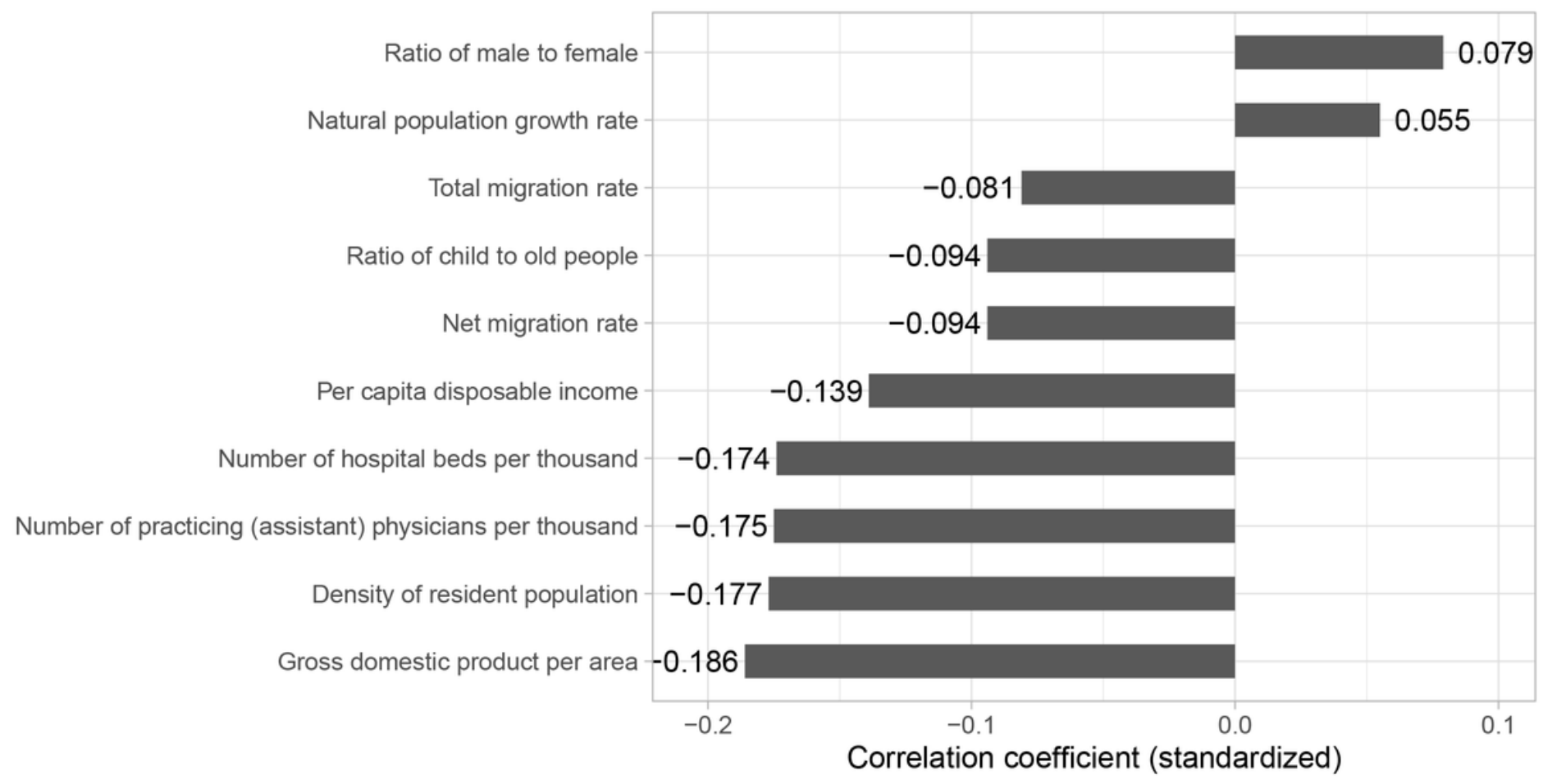

\section{Figure 5}

The direction and intensity of the effects of socio-economic, health services, population structure, and population dynamics on the gap between life expectancy and health-adjusted life expectancy

\section{Supplementary Files}


This is a list of supplementary files associated with this preprint. Click to download.

- Appendix.docx 